\title{
Desmoplastic Fibroblastoma in Maxillary Alveolar Bone Mimicking An Odontogenic Lesion: A Novel Case Report with Review of Literature
}

\author{
Zohreh JAAFARI-ASHKAVANDI', Mehdi YASAMIN SHIRAZI' ${ }^{2}$, Sepideh ASSAR' \\ 'Department of Oral and Maxillofacial Pathology, Shiraz Dental School, Shiraz University of Medical Sciences, SHIRAZ, IRAN \\ 2Department of Endodontics, Birjand Dental School, Birjand University of Medical Sciences, BIRJAND, IRAN
}

\begin{abstract}
Desmoplastic fibroblastoma (Collagenous fibroma) is a rare benign soft tissue tumor that disseminates widely and can be intramuscular or subcutaneously. There are seven reported cases in the oral cavity so far. We reported a novel case of collagenous fibroma that has been found in the anterior part of maxilla in a 58-year-old woman as an intraosseous radiolucent lesion that has been discovered during routine radiography. Histopathologically, spindle and stellate-shaped fibroblasts in a collagen-rich stroma as well as binucleated and multinucleated fibroblasts with prominent nucleoli were seen. Desmoplastic fibroblastoma could be found in jaw bones and excision of the lesion is curative, and no aggressive growth has been reported.
\end{abstract}

Key Words: Desmoplastic fibroblastoma, Collagenous fibroma, Intraosseous, Maxilla, Oral cavity

\section{INTRODUCTION}

Desmoplastic fibroblastoma (collagenous fibroma) is a rare benign soft tissue tumor that disseminates widely and can be intramuscular or subcutaneously that has been first described in $1995(1,2)$. This tumor usually affects men with the age range of 40 to 69 years, though it has also been reported from 16 to 81 years (2). The largest case series with 63 cases was published in 1998 by Miettinen and Fetsch (2). Involvement of different anatomic sites by this lesion, such as chest wall, orbital rim and genital areas were reported (35). The intraoral cases are very rare and Mesquita et al. has reported the first case in 2001. Parotid gland and palate are the most reported locations for intraoral lesions $(2,6-8)$.

Desmoplastic fibroblastoma is usually a firm mobile slow-growing painless mass that is well-circumscribed, round to oval, or lobulated (9). Microscopically, it shows a hypocellular soft tissue mass that is mostly composed of abundant collagen fibers. Tumor cells are spindle or stellate-shaped with large nuclei that may contain eosinophilic nucleoli. In dense fibrotic areas, the cells do not have any mitotic activity or necrosis (2). Binucleated cells and multinucleated giant cells may also be observed. Due to the surrounding collagenous stroma, few amounts of blood vessels were detected in this lesion. Inflammatory cells are usually absent $(1,2)$. By immunohistochemical staining, the cells show strongly positive immunoreactivity

(Turk Patoloji Derg 2018, 34:247-250)

Received : 08.01.2016 Accepted : 16.03 .2016 for vimentin and a variable immunoreactivity for smooth muscle actin $(10,11)$.

Herein we report a novel case of collagenous fibroma in the anterior part of maxilla and were unexpectedly presented as an intraosseous periapical lesion.

\section{CASE REPORT}

A 58-year-old woman was referred to the Dental School of Shiraz University of Medical Sciences because of an intraosseous radiolucent lesion that has been discovered during routine dental examination. The lesion was a wellcircumscribed, rounded radiolucency with a diameter of $20 \mathrm{~mm}$ in association with the root of right upper incisors. The patient did not report any pain or symptom and there were no swelling, redness or other changes on the overlying gingiva. Regional lymph nodes were normal on clinical examination. The patient's medical history was not remarkable. According to the vital test examination, adjacent teeth were diagnosed as vital but root canal therapy of tooth \#2 was done by a general dentist because of this radiolucent lesion, Three months after root canal therapy, the endodontist referred the patient for excisional biopsy because of no healing changes in the area (Figure 1). An excisional biopsy was done (Figure 2) and the lesion was sent to the Oral and Maxillofacial Pathology Department for histopathological examination. The formalin-fixed lesion was a piece of round creamy elastic soft tissue

Correspondence: Sepideh ASSAR

Department of Oral and Maxillofacial Pathology, Shiraz Dental School,

Shiraz University of Medical Sciences, SHIRAZ, IRAN

E-mail: sepideh1986@yahoo.com Phone: +98913 1932879 
measuring $1.3 \times 1 \times 1$ centimeters and was shiny on its cut surface.

Hematoxilyn\& eosin stained sections showed a benign, wellcircumscribed mesenchymal tumor consisting of spindle and stellate-shaped fibroblasts in a collagen rich stroma. Some fibroblasts were binucleated and multinucleated with prominent nucleoli. A few blood vessels were detected. Inflammatory cells, areas of necrosis, calcification, and

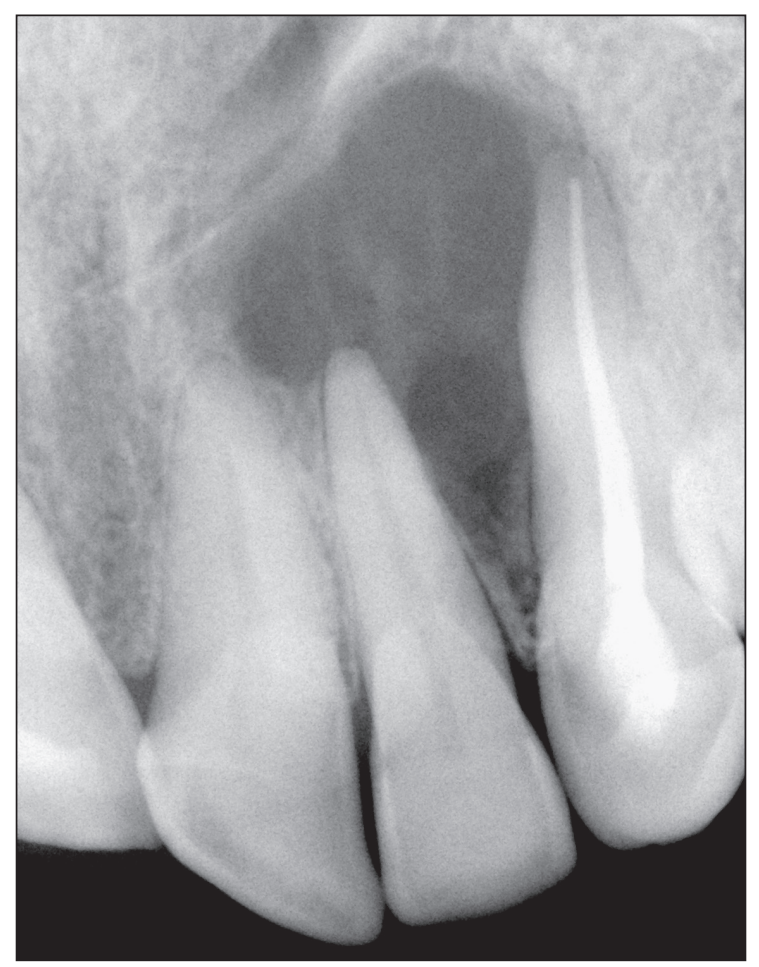

Figure 1: Periapical radiograph of the patient after root canal therapy: A well- defined radiolucent lesion mimicking periapical lesions.

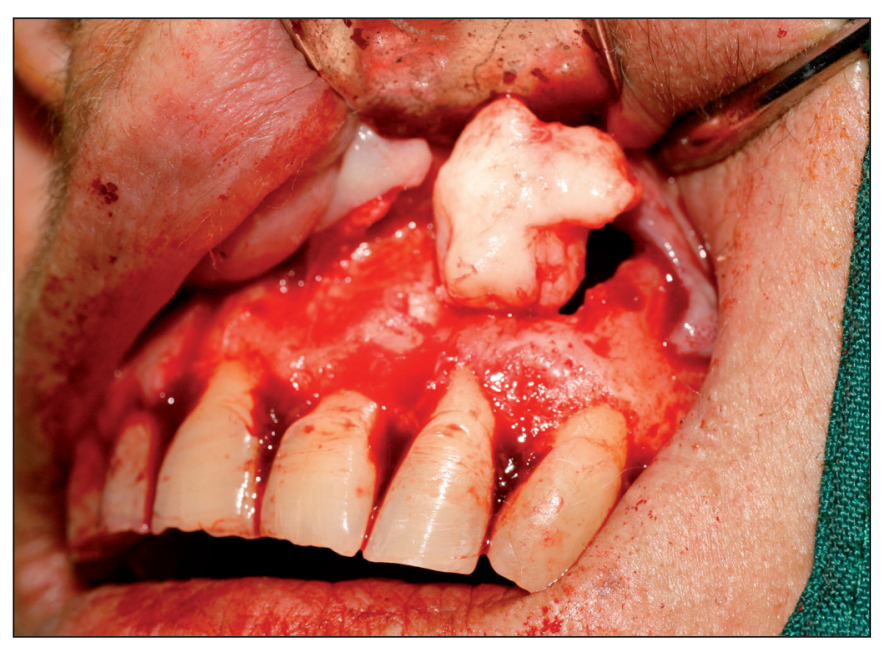

Figure 2: Excisional biopsy of the mass. mitosis were absent (Figure 3A,B). Masson's trichrome staining revealed abundunt collagen bundles (Figure 4). Immunohistochemical (IHC) examination was positive for vimentin (Figure 5A), SMA, CD34 and S-100 were negative (Figure 5B). According to these findings, a diagnosis of desmoplastic fibroblastoma was established. The patient was examined after 1 year and there was no recurrence of the lesion.

\section{DISCUSSION}

Desmoplastic fibroblastoma or collagenous fibroma is a benign fibroblastic/myofibroblastic tumor that typically occurs subcutaneously. No intraosseous lesion has been reported so far and the rare bony cases that have been reported demonstrated erosion on the surface of bone. Excision of the lesion is curative and no aggressive growth has been reported (12) .
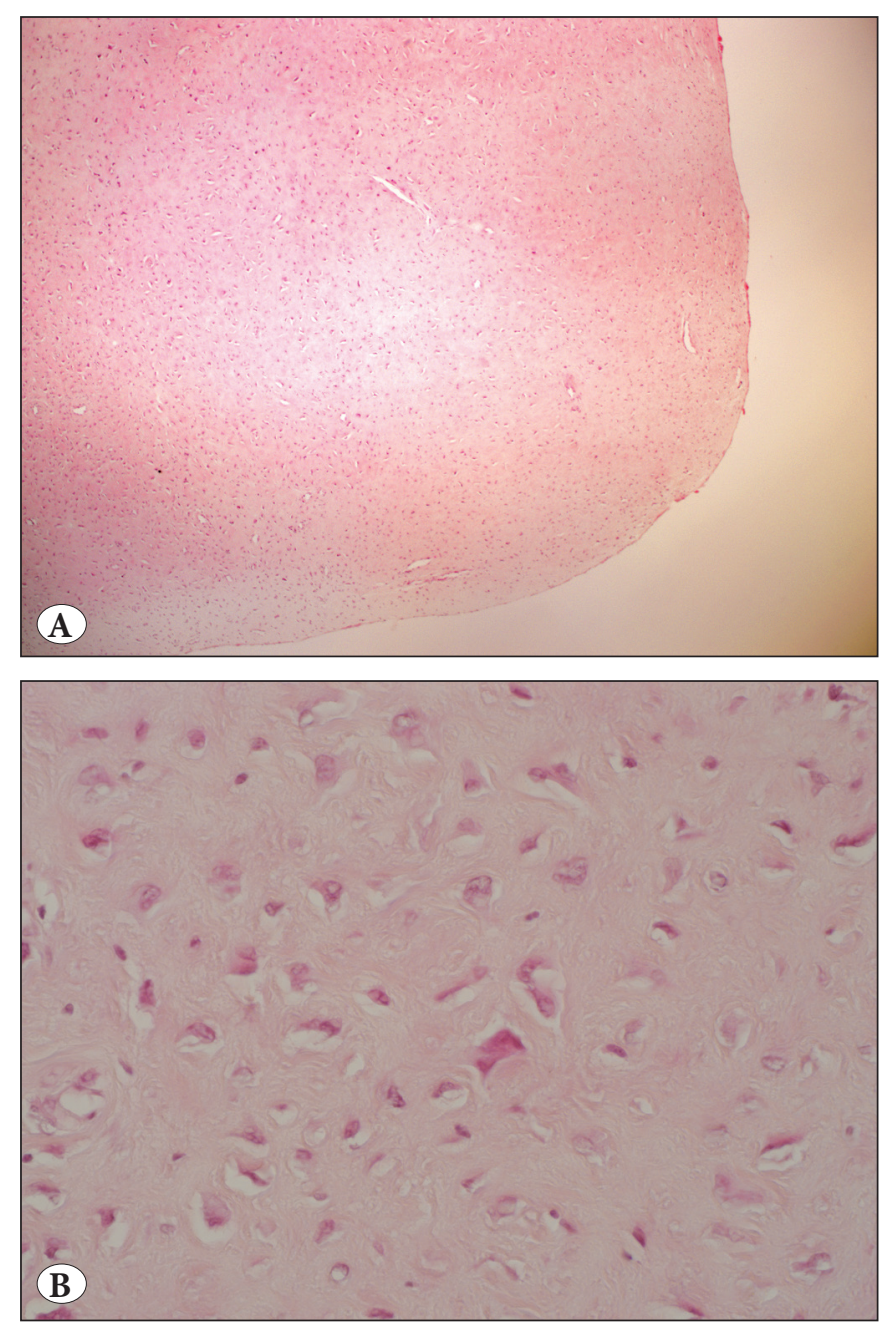

Figure 3: A) Well-circumscribed paucicellular mesenchymal tumor (H\&E; x40), B) Binucleated, stellate fibroblasts with prominent nucleoli (H\&E; $\mathrm{x} 400)$. 


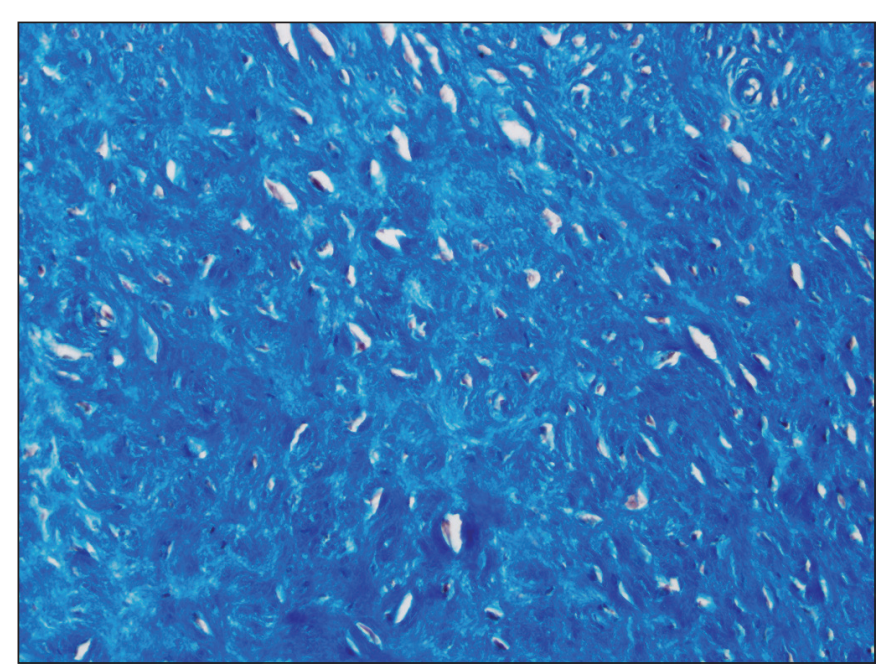

Figure 4: Stroma with abundant collagen bundles (Masson's trichrome; $\mathrm{x} 200)$.
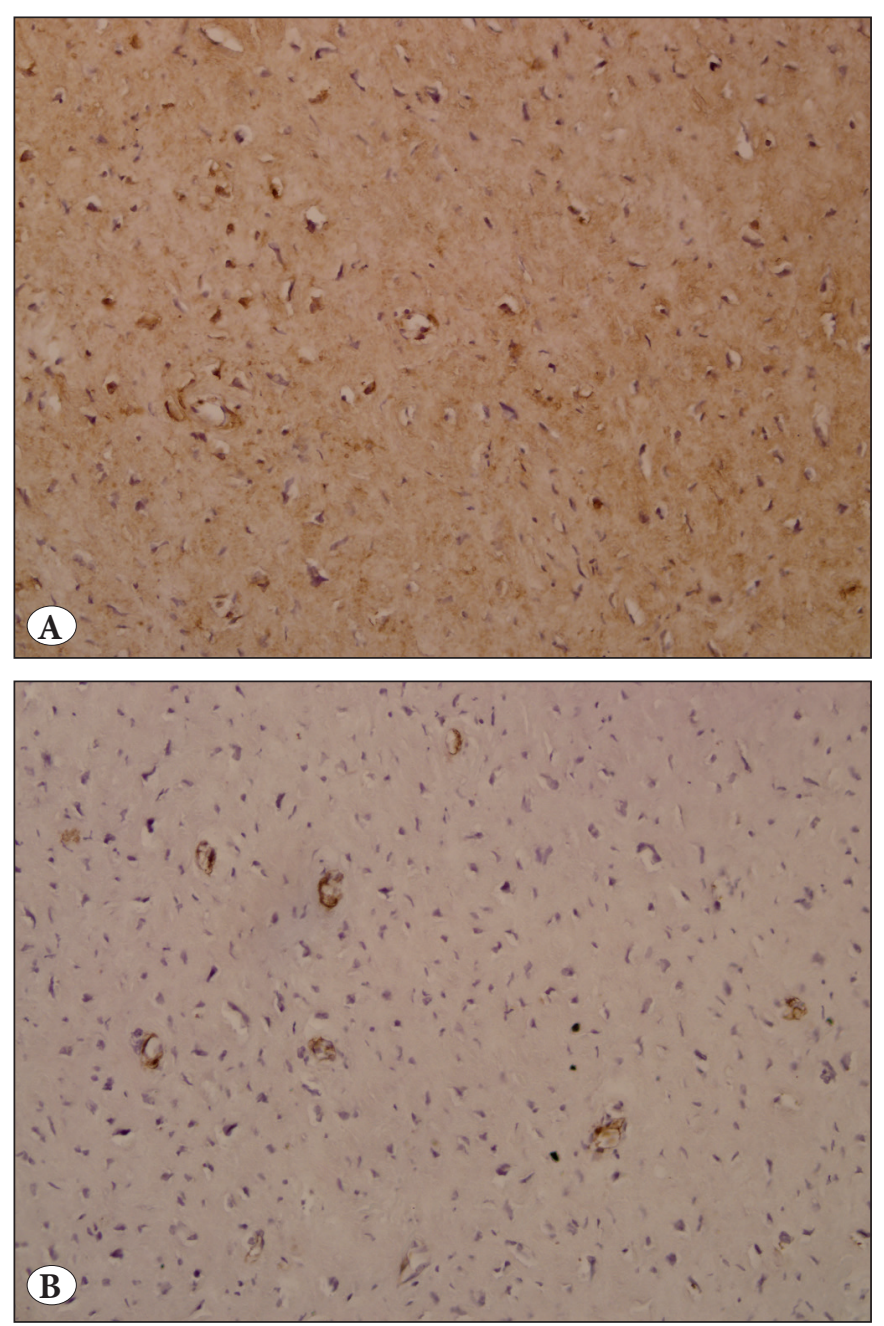

Figure 5: A) Spindle- and stellate-shaped cells positive for vimentin (IHC; x200), B) Negative for SMA (IHC; x200).
Seven cases have been reported in the oral cavity so far in the buccal mucosa, palate, parotid and gingiva. Our case is the first case that appeared to be intraosseous. After histopathological evaluation, a scar, desmoplastic fibroma, neurofibroma, chonromyxoid fibroma, lowgrade fibromyxoid sarcoma, calcifying fibrous- pseudo tumor and solitary fibrous tumor were considered in the differential diagnosis. There was no history of previous surgery or trauma that could cause a scar. Also there were no chondroid or myxoid area and no mitosis to prove chondromyxoid fibroma or fibromyxoid sarcoma. The lesion was hypocellular with no fascicular pattern so desmoplastic fibroma was ruled out and there was no calcification in the lesion for it to be a calcifying fibrous pseudo tumor. Neurofibroma has a myxoid stroma and spindle cells with wavy nuclei, which were not seen in our case. Negative S-100 protein and CD34 staining ruled out neurofibroma and solitary fibrous tumor.

Desmoplastic fibroblastoma is a tumor of soft tissue but Merriman et al. reported a case in which the tumor infiltrated and destructed the scapula (13).

This lesion is very rare in the oral cavity, Mesquita et al. has reported the first oral lesion in a 37-year-old woman. In their report, the histopathological features were similar to our case but clinically, in their case, the lesion was a solitary and firm $5 \mathrm{~cm}$ nodule in the palate and was not intraosseous. Histopathological sections showed stellate or spindle-shaped cells in a hypovascular fibrous stroma. Foci of fat were identified at the edges. Mitotic figures and tumor necrosis were absent. IHC staining was positive for vimentin, and a few cells were positive for alphasmooth muscle actin. Extracellular matrix of the tumor was immunopositive for type I and type III collagen and fibronectin (7).

Bhagalia et al. reported a 58-year-old female patient with the chief complaint of a firm, slow-growing, pedunculated lesion in the lingual gingival region in relation to the left $3^{\text {rd }}$ molar that had appeared in three months without any pain or teeth mobility. The connective tissue demonstrated dense collagen bundles with distribution of spindle to stellate fibroblastic cells. Multinucleated giant cells were also observed. Blood vessels and inflammatory cells were few in number. Fat cells were seen in the center of the section. Masson's trichrome staining revealed areas positive for collagen and some areas positive for muscle (11).

Immunohistochemical investigation can be for the definitive diagnosis and rule out other lesions in the differential diagnosis of desmoplastic fibroblastoma. We performed Masson's trichrome staining and immunohistochemical 
staining for vimentin, S-100 protein, SMA, and CD34. We found that collagen bundles were abundant by Masson's trichrome; the cells were strongly positive for vimentin and negative for S-100 protein, SMA and CD34. It is similar to the previous cases reported by Mesquita et al. (7).

De Sousa et al. in 2011 reported a 56-year-old man with a well-circumscribed, sessile, pink-colored mass in the left buccal mucosa. The mass was single, measuring $35 \mathrm{~mm}$ in diameter. Histopathologic features were similar to our case. Immunopositivity for vimentin and some positive cells with Factor XIIIa were seen (10).

In 2014, another case of oral cavity involvement has been reported in an 8-year-old boy with the complaint of a swelling in the left buccal mucosa in 6 months. In periapical radiography, there was no evidence of any periapical or other osteolytic lesions. Van Gieson special staining was positive for thick bundles of collagen (12).

Shimoyama et al. reported a case of desmoplastic fibroblastoma in a 49-year-old woman with a painless slowlygrowing polypoid palatal mass. Her medical history was significant for rheumatoid arthritis and she had taken prednisolone for 4 years. There was no evidence of root resorption or bone destruction in the radiography. Histopathologic features were similar to other reported cases. Thick collagenized desmoid-like areas were also seen focally. IHC was diffusely positive for vimentin and there were some positive cells for SMA (14). All of the cases mentioned above were excised with no recurrence during follow-up. The case discussed in our report is a 58 -year-old woman, although epidemiologic features of the previous studies point out to male predominance in the fifth to seventh decades of life (2). However, case reports in the oral cavity revealed female predominance. Most of the cases were found in sixth decades of life, and palate was the most common site of involvement. Buccal mucosa, gingiva and alveolar ridge have also been reported with less frequency.

The exact cause of this lesion is still unclear but some studies stated that it might develop due to a chromosomal rearrangement (11q12) that could also occur in the fibroma of the tendon sheath (15). Some authors considered this lesion as a neoplasm, which does not have a preceding traumatic or inciting factor $(1,16)$. The treatment of choice for this lesion is total surgical excision and the prognosis is excellent with no recurrence (7).

\section{ACKNOWLEDGMENT}

The authors thank the Vice-Chancellery of Shiraz University of Medical Science for supporting this research. The authors would like to thank Dr. Sh. Hamedani (DDS,
MSc) from the Dental Research Development Centre for helping with the English and editorial assistance in the manuscript.

\section{REFERENCES}

1. Evans HL. Desmoplastic fibroblastoma. A report of seven cases. Am J Surg Pathol. 1995;19:1077-81.

2. Miettinen M, Fetsch JF. Collagenous fibroma (desmoplastic fibroblastoma): A clinicopathologic analysis of 63 cases of a distinctive soft tissue lesion with stellate-shaped fibroblasts. Hum Pathol. 1998;29:676-82.

3. Stacy RC, Jakobiec FA, Fay A. Collagenous fibroma (desmoplastic fibroblastoma) of the orbital rim. Ophthal Plast Reconstr Surg. 2013;29:e101-4.

4. Park JS, Bae KH, Oh HK, Park JB, Park DH. Genital desmoplastic fibroblastoma (collagenous fibroma). Urol J. 2014;11:1849-51.

5. Kawaguchi Y, Fujita T, Hanaoka J. Invasive desmoplastic fibroblastoma in the chest wall. Ann Thorac Surg. 2015;99:e85-6.

6. Cazal C, Etges A, Almeida FC, de Souza SC, Nunes FD, de Araujo VC. Collagenous fibroma (desmoplastic fibroblastoma) of alveolar bone: A case report. J Br Pathol Med Lab 2005;41:185-8.

7. Mesquita RA, Okuda E, Jorge WA, de Araujo VC. Collagenous fibroma (desmoplastic fibroblastoma) of the palate: A case report. Oral Surg Oral Med Oral Pathol Oral Radiol Endod. 2001;91: 80-4.

8. Wilson C, Summerall J, Lubin J, Mesko TW. Collagenous fibroma (desmoplastic fibroblastoma): A unique presentation as a goiter in an 88-year-old man. Ann Diag Pathol. 2000;4:165-9.

9. Jham BC, De Mesquita Netto AC, Resende RG, Ribeiro DC, Mesquita RA. Pedunculated desmoplastic fibroblastoma (collagenous fibroma) of the oral cavity: A previously unreported clinical presentation. Int J Oral Maxillofac Pathol. 2013;4:21-6.

10. de Sousa SF, Caldeira PC, Grossmann Sde M, de Aguiar MC, Mesquita RA. Desmoplastic fibroblastoma (collagenous fibroma): A case identified in the buccal mucosa. Head Neck Pathol. 2011;5:175-9.

11. Bhagalia S, Jain M, Pardhe N, Sireesha SK. Collagenous fibroma (desmoplastic fibroblastoma) of the oral cavity. J Oral Maxillofac Pathol. 2012;16:277-9.

12. Varghese T, Pillai KS, Sarojini SB, Khosla E. Desmoplastic fibroblastoma (collagenous fibroma) in the oral cavity. J Indian Soc Pedo Prev Dent. 2014;32:238-41.

13. Merriman DJ, Deavers MT, Czerniak BA, Lin PP. Massive desmoplastic fibroblastoma with scapular invasion. Orthopedics. 2010;33.

14. Shimoyama T, Horie N, Ide F. Collagenous fibroma (desmoplastic fibroblastoma): A new case originating in the palate. Dentomaxillofac Radiol. 2005;34:117-9.

15. Sciot R, Samson I, van den Berghe H, Van Damme B, Dal Cin P. Collagenous fibroma (desmoplastic fibroblastoma): Genetic link with fibroma of tendon sheath? Mod Pathol. 1999;12:565-8.

16. Nielsen GP, O'Connell JX, Dickersin GR, Rosenberg AE. Collagenous fibroma (desmoplastic fibroblastoma): A report of seven cases. Mod Pathol. 1996;9:781-5. 